\title{
Dietary essential fatty acids and brain function: a developmental perspective on mechanisms
}

\author{
Patricia E. Wainwright \\ Department of Health Studies and Gerontology, University of Waterloo, Waterloo, Ontario N2L 5H1, Canada
}

\begin{abstract}
Brain development is a complex interactive process in which early disruptive events can have long-lasting effects on later functional adaptation. It is a process that is dependent on the timely orchestration of external and internal inputs through sophisticated intra- and intercellular signalling pathways. Long-chain polyunsaturated fatty acids (LCPUFA), specifically arachidonic acid and docosahexaenoic acid (DHA), accrue rapidly in the grey matter of the brain during development, and brain fatty acid (FA) composition reflects dietary availability. Membrane lipid components can influence signal transduction cascades in various ways, which in the case of LCPUFA include the important regulatory functions mediated by the eicosanoids, and extend to long-term regulation through effects on gene transcription. Our work indicates that FA imbalance as well as specific FA deficiencies can affect development adversely, including the ability to respond to environmental stimulation. For example, although the impaired water-maze performance of mice fed a saturated-fat diet improved in response to early environmental enrichment, the brains of these animals showed less complex patterns of dendritic branching. Dietary $n$-3 FA deficiency influences specific neurotransmitter systems, particularly the dopamine systems of the frontal cortex. We showed that dietary deficiency of $n$-3 FA impaired the performance of rats on delayed matching-to-place in the water maze, a task of the type associated with prefrontal dopamine function. We did not, however, find an association over a wider range of brain DHA levels and performance on this task. Some, but not all, studies of human infants suggest that dietary DHA may play a role in cognitive development as well as in some neurodevelopmental disorders; this possibility has important implications for population health.
\end{abstract}

Essential fatty acids: Arachidonic acid: Docosahexaenoic acid: Brain development: Animal models

Current theories of 'biological programming' in relation to the risk for the development of disease in adults emphasise the importance of a lifespan perspective. They describe a process whereby factors, such as undernutrition or exposure to drugs or hormones, during a sensitive developmental period result in long-lasting changes in the structure or function of specific organs or body systems (Godfrey \& Barker, 2000). Such factors may be particularly important in relation to the development of the brain, because of its integral role not only in maintaining physiological homeostasis, but also in initiating and coordinating adaptive behavioural responses. The present paper addresses the possible mechanisms whereby dietary long-chain polyun- saturated fatty acids (LCPUFA) may contribute to the development and maintenance of the functional capacities of the brain. It begins with a brief overview of the complex interactions of biological and environmental factors that constitute the underpinnings of brain development, including the ways in which nutritional factors can influence these processes. Next it addresses the relationship between dietary polyunsaturated fat content and alterations in brain fatty acid composition, and how these may translate into functional differences. Following discussion of our work related to these questions, the final section considers the evidence for a role of the $n$-3 LCPUFA, specifically docosahexaenoic acid (DHA), in the functional development of the brain.

\footnotetext{
Abbreviations: AA, arachidonic acid; DHA, docosahexaenoic acid; EFA, essential fatty acids; LCPUFA, long-chain polyunsaturated fatty acids; LNA, $\alpha$-linolenic acid; PG, prostaglandins; PKU, phenylketonuria.

Corresponding author: Professor Patricia E. Wainwright, fax +1 519746 2510, email wainwrig@ healthy.uwaterloo.ca
} 


\section{Brain development}

\section{Behavioural neuroscience}

The brain is not a homogeneous structure, and the timing and course of maturation varies considerably among brain regions (Rodier, 1980). In man the anatomical and functional specialisation of neurons proceeds from the last trimester until early adulthood, and it has been suggested that this protracted period of brain development may be the origin of human behavioural flexibility (Saugstad, 1998). The function of the brain depends ultimately on the operations of neural systems that are established by the patterns of synaptic connections among neurons. Behavioural neuroscience addresses the factors that, at an organismic level, influence the establishment of these neural systems. This endeavour cannot be accomplished independently of a developmental perspective, because events (both internal and external to the organism) that occur early in the development of the nervous system influence the final pattern of connection between nerve cells.

\section{Neurobiological development}

The sequence of events that constitute the development of the mammalian brain can be described briefly as follows (Nowakowski \& Hayes, 1999). The primitive neural tube is formed during the first 4 weeks in man, followed by a period of further cell proliferation and diversification into neurons and glia (5-18 weeks). At the same time there is neuronal migration from the ventricular germinal zone to the final position. Migration has a role in establishing the identity of some neurons and may define their functional properties and future connections. Differentiation of the neurons occurs over a span that encompasses the late prenatal period as well as the postnatal period. It comprises axonal and dendritic outgrowth, as well as the expression of enzymes and receptors for specific neurotransmitters. Myelination is a late-occurring process, starting in the third trimester and extending into the third decade and possibly beyond. During the last trimester there is also what is referred to as an 'exuberant' increase in the number of synaptic contacts. This process is followed by a series of regressive events, including programmed cell death (apoptosis) and synaptic and axonal pruning. These processes, which continue through the second decade of life, are influenced strongly by environmental input, and are thought to be the mechanisms underlying the establishment of circuitry that is functional in terms of the brain's adaptive capacity, including behaviour. Where it was thought previously that neurogenesis and synapse formation occur only during brain development, there is recent evidence of neuronal generation in adult mammalian brains, including those of primates (Gould et al. 1999).

\section{Role of early environment}

A psychobiological approach to the development of brain and behaviour describes a complex epigenetic process in which genes and environment interact as a system, where events at earlier stages can affect subsequent development through an intricate series of feedback and feedforward loops (Gottlieb, 1998). Evolutionary and ecological considerations are essential to this perspective, because species have evolved through natural selection to function optimally in their own ecological niche. Thus, the developmental outcomes normally associated with the speciestypical genome are also dependent on input from the species-typical environment. Although the genome may have considerable influence on the structural architecture of evolutionary older areas of the brain, the more modern structures, such as the cerebral cortex, show considerable plasticity, where the nature and timing of environmental input have considerable influences on the resulting organisational pattern (Damasio, 1994).

There are different theoretical perspectives on the role of the environment in the development of specific brain systems. The predominant theory, selectionism, postulates discrete stages, with intrinsic construction ("prerepresentation') followed by selective stabilisation of functionally meaningful connections. Neural constructivism, on the other hand, is more radical in postulating that structure derives ultimately from ongoing dynamic interactions between the mechanisms of neural growth and environmentally-derived neural activity (Quartz \& Sejnowski, 1997). There is nonetheless agreement on the essential role of neural activity, not only that generated in response to external stimulation, but also that generated internally by the developing nervous system itself, i.e. the organism's behavioural state. The modulation of behavioural state depends on the activity of the neurons located in the brainstem and basal forebrain which distribute neurotransmitters (e.g. noradrenaline, dopamine, serotonin, acetylcholine) to widespread regions of cortex and subcortical nuclei, and recent evidence supports the important contribution of these systems to the development of cortical organisation (Osterheld-Haas \& Hornung, 1996; Berger-Sweeney \& Hohmann, 1997). Thus, the neural systems subserving cognitive function do not develop independently, but as part of a complex interactive network. A further level of complexity is added when one considers that the homeostatic regulatory systems are themselves influenced by the early environment. Different patterns of maternal behaviour in rats, for example, are associated with differential responsiveness of the hypothalamo-pituitaryadrenal (HPA) axis in their offspring (Francis \& Meaney, 1999). Early handling, which engenders increased maternal-pup contact, results in attenuated fearfulness in a novel environment and decreased hypothalamo-pituitaryadrenal responses to a wide variety of stressors. These effects are related to changes in gene expression and differences in glucocorticoid regulation by the hippocampus and frontal cortex. They become more pronounced with ageing, and there is some evidence that individual differences in hippocampal function of this type may be associated with ageing-related cognitive decline (McEwan et al. 1999). Thus, the early environment is able to 'program' the stress response essential for adaptation and survival over the lifespan of the animal.

In discussing the integral role of experience in the development of the brain, Black (1998) distinguishes between two processes, one termed 'experience-expectant' and the other 'experience-dependent'. It is in relation to 
experience-expectant processes that the concept of sensitive periods is intrinsic, being defined as a time when the organism is maximally ready (primed) to receive appropriate stimulation. In the organisation of the visual system, for example, specific visual input during discrete developmental periods is necessary for the development of fully functional capacity (Shatz, 1992). Experiencedependent processes, on the other hand, optimise an individual's adaptation to specific and sometimes unique features of the environment. Although they are not seen as involving sensitive periods, there may be sequential dependencies, such that the capacity to respond to the environment is influenced by and related to those of preceding and subsequent stages of development. These interdependencies between the structure of the brain and the environment are illustrated in the extensive literature on the effects of environmental enrichment. Enriched housing, or training on specific tasks, is associated with structural changes in specific regions of the brain, including increased cortical thickness, acetylcholine levels, dendritic branching, number of synapses, as well as changes in glial cells; environmental enrichment also enhances performance on complex learning tasks (Black et al. 1998; Kolb et al. 1998; Klintsova \& Greenough, 1999). It is important to note that the ability to benefit from environmental stimulation is dependent not only on the animal's sensory and motor capacities, but also on its overall levels of activity or reactivity. Thus, in addition to direct biochemical effects on specific aspects of neuronal architecture or function, it is possible to affect the development of the brain indirectly through more global effects on regulatory function. Moreover, any factor that affects the ability of the animal to engage actively with the environment during critical developmental periods, as might be seen for example in situations of energy restriction or anaemia, can also affect development adversely by altering the nature of these essential interactions (Pollitt, 2001).

\section{Nutritional neuroscience}

There is a substantial literature that indicates that the chemistry and function of both the developing and mature brain can be influenced by the diet (Fernstrom, 2000). Direct effects on development are likely to be related to alterations in levels or timing of the various necessary growth factors, which include the neurotransmitters. In the adult nervous system neurotransmitters mediate intercellular communication by activating specific receptors and secondmessenger systems. In the developing system, however, they also function as trophic factors to regulate neurogenesis, neural migration and synaptogenesis (Lauder, 1993; Levitt et al. 1997). Many of these neurotransmitters are derived from nutrient precursors; tyrosine is the amino acid precursor of dopamine and noradrenaline, tryptophan of serotonin and choline of acetylcholine. In some cases they may also require the presence of micronutrient cofactors such as minerals and vitamins. Very often dietary components interact: this process is seen in the studies which show that the levels of tyrosine and tryptophan in the brain are related to the relative levels of protein and carbohydrate in the diet (Fernstrom, 2000). In fact, an interesting example of the effect of protein imbalance on brain development is seen in the condition termed phenylketonuria (PKU) in man. PKU results from a recessive mutation in the gene coding for phenylalanine hydroxylase, which converts phenylalanine to tyrosine, the precursor of dopamine. This condition leads to an excess of phenylalanine in the blood, which impairs mental development in infants. Although a diet low in phenylalanine started early in development can ameliorate much of the cognitive impairment, subtle cognitive deficits remain that are related specifically to the function of the dopaminergic neurons in the prefrontal cortex (Diamond et al. 1997). It is hypothesised that, despite the dietary treatment, the residual phenylalanine-tyrosine imbalance, which results in less tyrosine reaching the brain, is sufficient to affect the development of the particularly sensitive population of prefrontally-projecting dopamine neurons. Associated with PKU is a condition known as maternal PKU, where a pregnant woman with the genetic condition, but who has been treated effectively during development, has a genetically-normal infant who shows symptoms of PKU (Waisbren, 1999). This outcome is due to exposure of the fetus to abnormally high amounts of phenylalanine in utero, and illustrates that maternal nutrition is a factor that cannot be overlooked when considering nutritional effects on brain development.

There are two possible strategies in studying nutritional effects on behaviour. The first is based on the assumption that dietary-induced changes at a molecular or cellular level will necessarily have functional consequences. The challenge then becomes that of identifying the appropriate functional outcome to be measured. In the interests of hypothesis-driven enquiry, the best approach is to measure an outcome known to be associated with a particular biochemical pathway. For example, the literature on the role of the prefrontal dopamine system suggests that tests of short-term or 'working memory', i.e. memory relevant to solving a unique problem embedded in a particular task (as opposed to reference memory, which refers to learning the rules associated with the task generally) might prove particularly informative (Murphy et al. 1996). Hence the choice of delayed-matching type tasks in behavioural studies of PKU, where subjects are required to hold information in memory for a specified time period in order to make an appropriate subsequent choice. A potential weakness of this strategy is that of false negative findings, because the complexity and plasticity of the developmental pathways involved may well preclude finding the predicted association, although there may well be effects in other domains. This situation is where the second strategy may prove useful. Here the approach used is similar to that used in neurotoxicity testing, where comprehensive batteries of tests are used to assess performance across a wide range of behavioural outcomes. In addition to tests of sensory capacity and emotional reactivity, these batteries include various measures of learning and memory, as well as species-typical behavioural adaptations. Such test batteries are usually not based on a specific hypothesis, because they were designed as a screening tool. This approach, together with the number of tests they encompass, leads to a concern with respect to an increased probability of false positive findings. However, they may serve to direct attention to 
the possible neural systems involved, which can then be investigated further using a more systematic approach.

In contrast to emphasis of earlier research on the effects of undernutrition, i.e. overall energy and nutrient deficiency, there has been a renewed interest in the role of specific nutrients, e.g. choline or $\mathrm{Fe}$, on brain and behavioural development (Wauben \& Wainwright, 1999). The following section addresses the role of dietary lipids in this regard, specifically that of the LCPUFA.

\section{Dietary long-chain polyunsaturated fatty acids and brain development}

Lipids comprise 50-60\% of the dry weight of the adult brain, of which approximately $35 \%$ are in the form of LCPUFA, mainly arachidonic acid (20:4n-6; AA) and DHA (22:6n-3). These LCPUFA are derived through biosynthesis from their respective dietary essential fatty acid (EFA) precursors, linoleic acid $(18: 2 n-6)$ and $\alpha$-linolenic acid (18:3n-3; LNA), or they can be obtained directly from dietary sources such as eggs, fish and meat or, more recently, from single-cell oils. AA is found at relatively high levels in many tissues, whereas DHA is found at high levels in only a few tissues outside the central nervous system, such as the testes, but it represents a high proportion of the lipids of the retina and grey matter of the brain. AA and DHA accrue rapidly in the human brain during the third trimester and the early postnatal period, when the rate of brain growth is maximal. Currently, there is controversy over whether infant formulas that contain only linoleic acid and LNA are sufficient for optimum brain development, or whether additional preformed AA and DHA, as found in human milk, are necessary to ensure the long-term integrity of functional outcomes (for review, see Innis 2000; Lauritzen et al. 2001).

\section{Effects on membrane properties, second-messenger systems and gene expression}

Lipids have the potential to influence neuronal function in various ways (Jumpsen \& Clandidin, 1995). They can alter the physical properties of the membrane, and in this way influence a variety of membrane functions, including effects on ion channels and transport, endo- and exocytosis, and the activities of membrane-bound proteins. Since neurotransmission depends on membrane receptors, which in turn interact with $G$ protein and other second-messenger systems, alterations in membrane phospholipid-fatty acid composition may also affect the nature of these interactions. Furthermore, LCPUFA are released from membrane phospholipids by several classes of phospholipases and can themselves act as a source of second messengers in cellular signal transduction. Dietary fat also has profound effects on gene expression through binding directly with transcription factors, or through eicosanoid regulation of intracellular signalling cascades, which results in alterations in metabolism, growth and cell differentiation (Jump \& Clarke, 1999). There is some evidence of an association between the EFA content of the diet and changes in specific neurotransmitter systems in the rat brain. Chronic $n-3$ fatty acid deficiency, for example, reduced dopamine receptor binding and increased serotonin receptor density in the frontal cortex of young and aged rats; it also altered dopamine metabolism (Delion et al. 1994, 1996; Zimmer et al. 1998). Similarly, both serotonin and dopamine were decreased in the frontal cortex of piglets fed formula low in both linoleic acid and LNA (Innis \& de la Presa Owens, 1999). A subsequent study indicated an inverse relationship between DHA and dopamine in newborn rat brains; this study also demonstrated a relationship between dietary lipids and the fatty acid content of growth cones (Innis \& de la Presa Owens, 2001). These findings suggest that there may be specific developmental processes susceptible to the influence of dietary fatty acid deficiency, particularly those related to the frontal dopamine systems. In view of work on PKU discussed earlier, the effects on dopamine support the use of tests known to be sensitive to alterations in brain dopamine, such as the delayed matching tasks discussed earlier, when seeking behavioural correlates of dietary manipulations. Furthermore, dietary-induced changes in the fatty acid composition of growth cones may also influence brain plasticity. This effect may not only be relevant to the capacity of the developing brain to respond to environmental input, but may also influence the capacity of the mature brain to recover from injury.

\section{Long-chain polyunsaturated fatty acids as precursors of eicosanoids}

A major way in which LCPUFA can influence neuronal function is by acting as a source of the eicosanoids, a group of oxygenated $\mathrm{C}_{20}$ compounds, which includes prostaglandins (PG), thromboxanes, leukotrienes, and a variety of hydroxy and hydroperoxy fatty acids (for review, see Wainwright, 1997). Although AA is the major fatty acid precursor of the eicosanoids, these can also be derived from dihomo- $\gamma$-linolenic acid (20:3n-6), or eicosapentaenoic acid $(20: 5 n-3)$, and are referred to as series 1 (AA), 2 (dihomo- $\gamma$-linolenic acid) and 3 (eicosapentaenoic acid) respectively. The primary $\mathrm{PG}$ such as $\mathrm{PGD}_{2}, \mathrm{PGE}_{2}$ and $\mathrm{PGF}_{2}-\alpha$ play a direct role in neural activity, whereas other eicosanoids, such as thromboxane $\mathrm{A}_{2}$ and $\mathrm{PGI}_{2}$ are involved in regulating circulatory function. The various classes differ in terms of their functional effects, and there is considerable evidence that their production can be modified both qualitatively and quantitatively by changes in the relative proportions of the dietary $n-6$ and $n-3$ fatty acids (Kinsella et al. 1990). This finding may have important implications for the risk of damage from ischaemic and excitotoxic brain damage, as there is evidence for the involvement of LCPUFA and their derivatives in pathological events of this type (Siesjö \& Katsura, 1992). There is also a body of evidence implicating PG in the regulation of sleep-wake cycles (Hayaishi, 1991). A recent study indicated that supplementation of the maternal diet with DHA resulted in a more mature organization of sleep patterns in 1-d-old infants, thereby supporting a role for the EFA in these processes (C Lammi-Keefe, personal communication). The eicosanoids have also been implicated as key players in the integrated set of physiological responses, mediated by the cytokines, that the body mounts in response to tissue injury or infection, and which are accompanied by a constellation 
of behavioural changes that are referred to collectively as 'sickness behaviour' (Kent et al. 1992). The resemblance of this constellation of behavioural changes to the symptoms characteristic of clinical depression has provided further impetus for the work that addresses the possible involvement of the immune system in behavioural pathology.

\section{Effects of long-chain polyunsaturated fatty acids on brain growth, fatty acid composition and behaviour}

The focus of the present section is on work done in our laboratory in which we have used animal models to address the relationship between dietary fatty acid composition, brain growth and fatty acid composition, and behaviour. Severe combined deficiency of both $n-3$ and $n-6$ fatty acids, i.e. EFA deficiency, results in aberrant physical and behavioural development (for review, see Wainwright, 1992). We showed in mice that not only EFA deficiency, but also an extreme dietary fatty acid imbalance, in terms of high levels of DHA with low levels of $n-6$ fatty acids, also resulted in growth retardation, accompanied by impaired swimming ability in the Morris water maze, unless a portion of the $n-6$ fatty acids were provided in the form of AA (Wainwright et al. 1997). A subsequent study showed that the growth retardation was accompanied by behavioural retardation of a magnitude equivalent to that resulting from moderate undernutrition (Wainwright et al. 1999). These effects may be related to the role played by AA in the signalling actions of neuroendocrine cells, where there is evidence for its involvement in the release of both growth hormone and prolactin (Roudbaraki et al. 1996). The message to be taken from this information is that not only absolute, but also relative, dietary fatty acid content is important to developmental status. This factor is particularly important to keep in mind as the technology of food production changes, as seen in genetically-engineered oils with very much higher concentrations of specific biologically-active fatty acids than have been available until now.

We also investigated whether dietary fatty acid composition would influence the animal's ability to respond to environmental stimulation. In the first study, starting at conception, mice were fed one of three diets: a saturated-fat diet deficient in both $n-6$ and $n-3$ fatty acids; a diet deficient in $n$-3 fatty acids only; a control diet with $n-6: n-3$ fatty acids of approximately 4:1 (Wainwright et al. 1994a). Their offspring were raised in either enriched or standard environments for 6 weeks. The enriched environment constituted housing mice in groups of twelve in large cages with food and bedding, as well as varied opportunities for exploration and physical activity, such as digging trays, rotating series of toys and other manipulandae, e.g. assorted bedding materials. The standard environment constituted housing mice in pairs in standard opaque plastic mouse cages with only food and bedding. The mice were then tested for place learning on the Morris water maze. Animals fed the saturated-fat diet showed retarded growth and were impaired in their ability to learn the location of the platform; these deficits were unrelated to differences in swimming capacity or motivation. They also had the lowest levels of
DHA in the brain. The growth of the mice fed the $n-3$ fatty acid-deficient diet was unaffected, and their learning performance did not differ significantly from either of the other two groups. Contrary to the prediction that the group fed the deficient diets would be unable to benefit from environmental enrichment, maze performance was enhanced in all the enriched groups. However, we did show in a subsequent study that mice fed the saturated-fat diet and raised in an enriched environment showed less dendritic branching in pyramidal cells of occipital cortex than mice fed the control diet. (Wainwright et al. 1998a).

Although dietary deficiency of $n-3$ fatty acids during development does not appear to affect growth, it does lead to characteristic changes in brain fatty acid composition which include a decrease in DHA and a reciprocal increase in $22: 5 n-6$. There is also a reciprocal relationship between levels of dietary AA and DHA and those in the brain. The artificial-rearing model in rats, in which a rat-milk substitute is fed to neonatal rats via a gastrostomy tube, allows the researcher complete control over the dietary intake of infant rats during the period encompassing the brain growth spurt. We have used this model to conduct a series of studies in which we addressed the relationship between dietary levels of DHA and AA systematically by supplementing these fatty acids in varying amounts to diets with normal levels of linoleic acid and LNA (10 and $1 \mathrm{~g} / 100 \mathrm{~g}$ total fatty acids respectively). The first study using a $3 \times 3$ study design with $0,0.5$ and $2.4 \mathrm{~g} \mathrm{AA}$ and DHA/100 $\mathrm{g}$ total fatty acids showed that, because of the reciprocal relationship between dietary DHA and AA on tissue levels, if some of the $n-3$ fatty acids were provided as DHA, it was necessary to also provide a portion of the $n-6$ fatty acids as AA in order to attain a normal brain fatty acid composition (Ward et al. 1998). Dietary supplementation resulted in a wide range of brain AA and DHA relative to the unsupplemented group (AA and DHA at 0 and $0 \mathrm{~g} / 100 \mathrm{~g}$ total fatty acids respectively). The range of AA in forebrain phospholipids on day 18 was 86-110 g/100 g and that for DHA was 82-142 g/100 g; for the cerebellum the corresponding values were 75-122 and $77-153 \mathrm{~g} / 100 \mathrm{~g}$. There was also a non-linear relationship between DHA in brain and erythrocyte phospholipids such that there was a levelling off of brain levels at the higher levels of DHA in the blood. This finding would lead one to expect that functional outcomes might show similar doseresponse effects, such that the largest differences would be between animals fed deficient and adequate diets, with little or no variation above the threshold that could be said to define an adequate diet. This type of outcome is supported by our recent work, where, despite the substantial range of DHA attained in the brain, a subsequent behavioural study showed no apparent relationship between brain DHA and delayed matching-to-place in the Morris water maze (Wainwright et al. 1999). However, rats that were fed a marginal-EFA diet (Wainwright et al. 1999) or $n$-3 fatty acid-deficient diet (Wainwright et al. 1998b) were impaired on this task. In contrast, we were unable to show effects of three generations of dietary $n-3$ fatty acid deficiency on place learning in the water maze, despite a $50 \%$ reduction in brain DHA (Wainwright et al. 1994b), suggesting that the effect may be specific to short-term memory. 


\section{Specific role of dietary docosahexaenoic acid in human neural development}

There is a concern that at particular times during the lifespan, particularly during early development, the metabolic capacity to convert LNA to DHA may prove inadequate in man and thus provision of DHA in the diet may be essential, hence the term 'conditional essentiality' (Cunnane, 2000). There have been a number of reports showing that dietary $n-3$ fatty acid deficiency during development is associated with changes in various aspects of visual function in different species, including human infants (for review, see Carlson \& Neuringer, 1999; Innis, 2000; Lauritzen et al. 2001). On this basis, it has been suggested that DHA may play a unique role in the function of excitable membranes. Chronic dietary deficiency of LNA in animals has been associated not only with retinal changes, but also with alterations in performance on some behavioural tests of learning and memory (for review, see Reisbick \& Neuringer, 1997; Wainwright, 1997; Carlson \& Neuringer, 1999), leading some authors to infer cognitive effects. Although an emphasis on learning is defensible on the basis of a desire to identify nutritional factors that may have an impact on the development of human cognitive capacity, there are various reasons to be cautious in this respect. First, these findings are not consistent across laboratories. Second, the interpretation of some studies fail to take into account that alterations in non-cognitive functions (emotionality, arousal) or inadequate sensory and motor skills may be possible alternative explanations for their findings. These factors may not be viewed as problematic if one believes that, because it is performance that ultimately counts, to be able to demonstrate an effect on performance is sufficient. However, in order to understand the phenomenon fully, elimination of these potential confounds is essential. Moreover, implicit in much of this work has been the assumption that learning and memory are unitary phenomena in terms of brain function. However, there is evidence that different neural systems are involved in different types of memory and that experimental manipulations that impair performance on one type of learning task may actually lead to an enhancement on another (Everitt \& Robbins, 1997). Thus, it is important to consider the functional domain that is being tapped by a particular task and to assess the outcome over a variety of tasks before drawing the conclusion that overall behavioural adaptation is better or worse.

There are several recent studies in which human infants have been assigned randomly to be fed formulas supplemented with DHA or with both DHA and AA, and assessed on cognitively-related measures, including the Bayley scales of development and visual recognition memory as assessed by the Fagan (1990) Infantest. Although there were no effects on visual recognition, preterm infants fed DHAsupplemented diets showed shorter look durations (Carlson \& Werkman, 1996; Werkman \& Carlson, 1996). Interestingly, this effect has also been reported in rhesus monkeys and it has been suggested that the longer look durations associated with lower DHA status may be due to an inability to shift attention from a visual stimulus (Reisbick et al. 1997). Although measures of visual recognition in infants do correlate moderately with later tests of childhood intelligence, the predictive validity of look duration during the choice trial has not yet been established (Colombo, 1997).

Other cognitive measures have also been used. A study in term infants, using a well-controlled task, showed improved problem solving at 10 months in infants fed for 4 months with formula supplemented with AA and DHA compared with those fed a control formula; the control was, however, very low in $n-3$ fatty acids (Willatts et al. 1998). In contrast, lower language scores were reported in 14-month-old term infants fed formula supplemented with DHA (Scott et al. 1998). However, these effects appear to have been transient and the predictive validity of early language with respect to later cognitive function is controversial (Carlson \& Neuringer, 1999). Recently, there have been reports from two randomised clinical studies in which the formula of term infants was supplemented with DHA and AA during the first postnatal months and development assessed at 18 months using the Bayley scales. One was a large multicentre trial in which there were no significant effects (Lucas et al. 1999). In the other smaller study the group of infants supplemented with DHA + AA showed a seven-point increase on the mental development index relative to controls (Birch et al. 2000). At its inception this study also included a breast-fed group, but 18-month data were not provided for this group for comparison. One concern with the use of the Bayley scales is that, although they may be useful in clinical assessment, there is little correlation in a normal population between these scores and later tests used to assess intelligence in school-aged children. Thus, the results of future more specific cognitive tests on this study population should prove informative.

\section{Possible involvement of long-chain polyunsaturated fatty acids in neurodevelopmental disorders}

The studies described earlier were designed to ascertain whether the addition of DHA and AA to infant formulas is advisable in the interests of promoting normal brain development. However, a role for LCPUFA has also been suggested in relation to schizophrenia and attention-deficit hyperactivity disorder, both conditions that have been characterised as neurodevelopmental disorders, where the symptoms are reflective of disruptions in the normal developmental pathways (Keshavan \& Hogarty, 1999; Taylor, 1999). It has been proposed that the changes in neurotransmitter function that have been associated with psychiatric conditions of this type may in some cases be secondary to changes in the lipid composition of neuronal phospholipids (Horrobin, 1999). The supporting evidence for this proposal in human subjects is largely indirect. It includes reports of increased phospholipase $\mathrm{A}_{2}$ activity in individuals with schizophrenia and, consistent with this finding, reduced levels of AA and DHA in erythrocyte membranes. It has also been reported that some children who have been diagnosed with attention-deficit hyperactivity disorder show alterations in tissue fatty acid profiles (Stevens et al. 1996). Related to this finding is the observation that the spontaneously-hypertensive rat, which 
is a commonly-used animal model of attention-deficit hyperactivity disorder (Sagvolden, 2000), differs in similar ways (Mills \& Huang, 1992). Moreover, there are some epidemiological and clinical studies that show an association between depression and a deficiency of $n-3$ LCPUFA (Hibbeln, 1999). Also noteworthy are reports of case studies where DHA supplementation has been shown to have a beneficial effect in patients with Zellwegger's syndrome, a peroxisomal disorder associated with severe retardation (Martinez, 1996). It will be very interesting, therefore, to see the outcomes of the more extensive studies on these questions that are currently underway.

\section{Conclusions}

Brain development is a complex interactive process in which early disruptive events, including an inappropriate supply of nutrients, can have far-reaching consequences. LCPUFA, particularly AA and DHA, are integral components of neuronal membranes. Alterations in membrane lipid components can influence crucial intra- and intercellular signalling pathways in various ways. Deficiencies of EFA influence specific neurotransmitter systems in animals, particularly the dopamine systems of the frontal cortex, as well as associated behaviours. There is some evidence that DHA may provide some advantage with respect to mental development, as assessed by standard infant developmental scales; future longitudinal studies should include more sensitive cognitive measures. These issues are important to resolve, because small changes at an individual level can have important implications for population health.

\section{Acknowledgements}

This work is supported by the Natural Sciences and Engineering Research Council of Canada; additional financial support for some of the studies was provided by Ross Laboratories and Martek Biosciences. The author is indebted to her collaborators, particularly Dr Y.-S. Huang, G. Ward and H.-C. Xing, and also to Ms D. McCutcheon for her invaluable contribution in terms of animal care. The energies and talents of dedicated graduate and undergraduate students is also much appreciated. The comments of Ms S. Giesbrecht and Mr T. Girard on an earlier version of this manucript were most helpful.

\section{References}

Berger-Sweeney J \& Hohmann CF (1997) Behavioral consequences of abnormal cortical development: insights into developmental disabilities. Behavioural Brain Research 86, 121-142.

Birch EE, Garfield S, Hoffman DR, Uauy R \& Birch DG (2000) A randomized controlled trial of early dietary supply of long-chain polyunsaturated fatty acids and mental development in term infants. Developmental Medicine and Child Neurology 42, 174-181.

Black JE (1998) How a child builds its brain: some lessons from animal studies of neural plasticity. Preventive Medicine 27, $168-171$.
Black JE, Jones TA, Nelson CA \& Greenough WT (1998) Neural plasticity and the developing brain. In The Handbook of Child and Adolescent Psychiatry, vol. 6, pp. 31-53 [N Alessi, JT Coyle, SI Harrison and S Eth, editors]. New York: John Wiley \& Sons.

Carlson SE \& Neuringer M (1999) Polyunsaturated fatty acid status and neurodevelopment: a summary and critical analysis of the literature. Lipids 34, 171-178.

Carlson SA \& Werkman SH (1996) A randomized trial of visual attention of preterm infants fed docosahexaenoic acid until two months. Lipids 31, 85-90.

Colombo J (1997) Individual differences in infant cognition: methods, measures and models. In Developing Brain and Behaviour: The Role of Infant Formula, pp. 339-385 [J Dobbing, editor]. London: Academic Press.

Cunnane SC (2000) The conditional nature of the dietary needs for polyunsaturates: a proposal to reclassify 'essential fatty acids' as 'conditionally-indispensable' or 'conditionallydispensable' fatty acids. British Journal of Nutrition $\mathbf{8 4}$, 803-812.

Damasio A (1994) Descartes Error: Emotion, Reason and the Human Brain. New York: Avon Books.

Delion S, Chalon S, Guillotaeu D, Besnard J-C \& Durand G (1996) $\alpha$-Linolenic acid deficiency alters age-related changes of dopaminergic and serotonergic neurotransmission in the rat frontal cortex. Journal of Neurochemistry 66, 1582-1591.

Delion S, Chalon S, Herault J, Guilloteau D, Besnard JC \& Durand G (1994) Chronic $\alpha$-linolenic acid deficiency alters dopaminergic and serotonergic neurotransmission in rats. Journal of Nutrition 124, 2466-2476.

Diamond A, Prevor MB, Callender G \& Druin DP (1997) Prefrontal cortex cognitive deficits in children treated early and continuously for PKU. Monographs of the Society for Research in Child Development 62, 1-208.

Everitt BJ \& Robbins TW (1997) Central cholinergic systems and cognition. Annual Review of Psychology 48, 649-684.

Fagan JF (1990) The paired-comparison paradigm and infant intelligence. Annals of the New York Academy of Sciences $\mathbf{6 0 8}$ 337-364.

Fernstrom J (2000) Can nutrient supplements modify brain function? American Journal of Clinical Nutrition 71, Suppl., 1669S-1673S.

Francis DD \& Meaney MJ (1999) Maternal care and the development of stress responses. Current Opinion in Neurobiology 9, 128-134.

Godfrey KM \& Barker DJP (2000) Fetal nutrition and adult disease. American Journal of Clinical Nutrition 71, 1344S-1352S.

Gottlieb G (1998) Normally occurring environmental and behavioral influences on gene activity: from central dogma to probabilistic epigenesis. Psychological Review 105, 792-802.

Gould E, Reeves AJ, Graziano MSA \& Gross G (1999) Neurogenesis in the neocortex of adult primates. Science 286, 548-552.

Hayaishi O (1991) Molecular mechanisms of sleep wake regulation: roles of prostaglandins $\mathrm{D}_{2}$ and $\mathrm{E}_{2}$. FASEB Journal 5, 2575-2581.

Hibbeln JR (1999) Long-chain polyunsaturated fatty acids in depression and related conditions. In Phospholipid Spectrum Disorder in Psychiatry, pp. 195-210 [M Peet, I Glen and DF Horrobin, editors]. Carnforth, Lancs.: Marius Press.

Horrobin DF (1999) The phospholipid concept of psychiatric disorders. In Phospholipid Spectrum Disorder in Psychiatry, pp. 3-20 [M Peet, I Glen and DF Horrobin, editors]. Carnforth, Lancs.: Marius Press.

Innis SM (2000) The role of dietary $n-6$ and $n-3$ fatty acids in the developing brain. Developmental Neuroscience 22, 474-480.

Innis SM \& de la Presa Owens S (1999) Docosahexenoic and arachidonic acid prevent a decrease in dopaminergic and 
serotonergic neurotransmitters in frontal cortex caused by a linoleic and $\alpha$-linolenic deficient diet in formula-fed piglets. Journal of Nutrition 129, 2088-2093.

Innis SM \& de la Presa Owens S (2001) Dietary fatty acid composition in pregnancy alters neurite membrane fatty acids and dopamine in newborn rat brain. Journal of Nutrition 131, 118-122.

Jump DB \& Clarke SD (1999) Regulation of gene expression by dietary fat. Annual Review of Nutrition 19, 63-90.

Jumpsen J \& Clandinin MT (1995) Brain Development: Relationship to Dietary Lipid and Lipid Metabolism. Champaign, IL: AOCS Press.

Kemperman G \& Gage FH (1999) New nerve cells for the adult brain. Scientific American 280, 48-53.

Kent S, Bluthe RM, Kelley KW \& Dantzer R (1992) Sickness behaviour as a new target for drug development. Trends in Pharmacological Sciences 13, 24-28.

Keshavan MS \& Hogarty GE (1999) Brain maturational processes and delayed onset in schizophrenia. Development and Psychopathology 11, 525-543.

Kinsella JE, Broughton KS \& Whelan JW (1990) Dietary unsaturated fatty acids: interactions and possible needs in relation to eicosanoid synthesis. Journal of Nutritional Biochemistry 1, 123-141.

Klintsova AY \& Greenough WT (1999) Synaptic plasticity in cortical systems. Current Opinion in Neurobiology 9, 203-208.

Kolb B, Forgie M, Gibb R, Gorny G \& Rowntree S (1998) Age, experience and the changing brain. Neuroscience and Biobehavioral Reviews 22, 143-159.

Lauder JM (1993) Neurotransmitters as growth regulatory signals: Role of receptors and second messengers. Trends in Neurosciences 16, 233-240.

Lauritzen L, Hansen HS, Jørgensen MH \& Michaelsen KF (2001) The essentiality of long chain $n-3$ fatty acids in relation to development and function of the brain and retina. Progress in Lipid Research 40, 1-94.

Levitt P, Harvey JA, Friedman E, Simansky K \& Murphy EH (1997) New evidence for neurotransmitter influences on brain development. Trends in Neurosciences 20, 269-274.

Lucas A, Stafford M, Morley R, Abbott R, Stephenson T, MacFadyen U, Elias-Jones A \& Clements H (1999) Efficacy and safety of long-chain polyunsaturated fatty acid supplementation of infant-formula milk: a randomised trial. Lancet 354, 1948-1954.

McEwen BS, de Leon MJ, Lupien S \& Meaney MJ (1999) Corticosteroids, the aging brain and cognition. Trends in Endocrinology and Metabolism 10, 92-96.

Martinez M (1996) Docosahexaenoic acid therapy in docosahexaenoic acid-deficient patients with disorders of peroxisomal biogenesis. Lipids 31, S145-S152.

Mills DE \& Huang Y-S (1992) Metabolism of $n-6$ and $n-3$ polyunsaturated fatty acids in normotensive and hypertensive rats. In Essential Fatty Acids and Eicosanoids, pp. 345-348 [A Sinclair and R Gibson, editors]. Champaign, IL: AOCS.

Murphy BL, Arnsten AFT, Goldman-Rakic PS \& Roth RH (1996) Increased dopamine turnover in the prefrontal cortex impairs spatial working memory performance in rats and monkeys. Proceedings of the National Academy of Sciences USA 93, 1325-1329.

Nowakowski RS \& Hayes NL (1999) CNS development: An overview. Development and Psychopathology 11, 395-417.

Osterheld-Haas MC \& Hornung JP (1996) Laminar development of the mouse barrel cortex: Effects of neurotoxins against monoamines. Experimental Brain Research 110, 183-195.

Pollitt E (2001) The developmental and probabilistic nature of the functional consequences of iron-deficiency anemia in children. Journal of Nutrition 131, 669S-675S.
Quartz SR \& Sejnowski TJ (1997) The neural basis of cognitive development: a constructivist manifesto. Behavioral and Brain Science 20, 537-596.

Reisbick S \& Neuringer M (1997) Omega-3 fatty acid deficiency and behavior: a critical review and future directions for research. In Handbook of Essential Fatty Acid Biology: Biochemistry, Physiology and Behavioral Neurobiology, pp. 397-426 [S Yehuda and DI Mostofsky, editors]. Totowa, NJ: Humana Press.

Reisbick S, Neuringer M, Gohl E, Wald R \& Anderson GJ (1997) Visual attention in infant monkeys: effects of dietary fatty acids and age. Developmental Psychology 33, 387-395.

Rodier PM (1980) Chronology of neuron development: animal studies and their clinical implications. Developmental Medicine and Child Neurology 22, 525-545.

Roudbaraki MM, Droulhault R, Bacquarty T \& Vacher P (1996) Arachidonic acid-induced hormone release in somatotropes: involvement of calcium. Neuroendocrinology 63, 244-256.

Sagvolden T (2000) Behavioral validation of the spontaneously hypertensive rat (SHR) as an animal model of attention deficit/hyperactivity disorder AD/HD. Neuroscience and Biobehavioral Reviews 24, 31-39.

Saugstad LF (1998) Cerebral lateralisation and rate of maturation. International Journal of Psychophysiology 28, 37-62.

Scott DT, Janowsky JS, Carroll RE, Taylor JA, Auestad N \& Montalto M (1998) Formula supplementation with long-chain polyunsaturated fatty acids: are there developmental benefits? Pediatrics 102, E59.

Shatz C (1992) The developing brain. Scientific American 267, 61-67.

Siesjö BK \& Katsura K (1992) Ischemic brain damage: focus on lipids and lipid mediators. In Neurobiology of Essential Fatty Acids, pp. 41-44 [NG Bazan, MG Murphy and G Toffano, editors]. New York: Plenum Press.

Stevens LJ, Zentall SS, Abate ML, Kuczek T \& Burgess JR (1996) Omega-3 fatty acids in boys with behavior, learning and health problems. Physiology and Behavior 59, 915-920.

Taylor E (1999) Developmental neuropsychopathology of attention deficit and impulsiveness. Development and Psychopathology 11, 607-628.

Wainwright PE (1992) Do essential fatty acids play a role in brain and behavioral development? Neuroscience and Biobehavioral Reviews 16, 193-205.

Wainwright PE (1997) Essential fatty acids and behaviour: is there a role for the eicosanoids? In Handbook of Essential Fatty Acid Biology: Biochemistry, Physiology and Behavioral Neurobiology, pp. 299-341[S Yehuda and DI Mostofsky, editors]. Totowa, NJ: Humana Press.

Wainwright PE, Bulman-Fleming MB, Lévesque S, Mutsaers L \& McCutcheon D (1998a) Saturated-fat diet during development alters dendritic growth in mouse brain. Nutritional Neuroscience 1, 49-58.

Wainwright PE, Huang Y-S, Bulman-Fleming B, Lévesque S \& McCutcheon D (1994a) The effects of dietary fatty acid composition combined with environmental enrichment on brain and behavior in mice. Behavioural Brain Research 60, 125-136.

Wainwright PE, Huang Y-S, Coscina DV, Lévesque S \& McCutcheon D (1994b) Brain and behavioral effects of dietary $(n-3)$ deficiency in mice: A three generation study. Developmental Psychobiology 27, 467-487.

Wainwright PE, Xing H-C, Girard T, Parker L \& Ward GR (1998b) Effects of dietary $n-3$ deficiency on amphetamine-conditioned place preference and working memory in the Morris water-maze. Nutritional Neuroscience 4, 281-293.

Wainwright PE, Xing H-C, Mutsaers L, McCutcheon D \& Kyle D (1997) Arachidonic acid offsets the effects on mouse brain and 
behavior of a diet with a low (n-6):(n-3) ratio and very high levels of docosahexaenoic acid. Journal of Nutrition 127, 184-193.

Wainwright PE, Xing H-C, Ward GR, Huang Y-S, Bobik E, Auestad N \& Montalto M (1999) Water maze performance is unaffected in artificially reared rats fed diets supplemented with arachidonic acid and docosahexaenoic acid. Journal of Nutrition 129, 1079-1089.

Waisbren SE (1999) Developmental and neuropsychological outcome in children born to mothers with phenylketonuria. Mental Retardation and Developmental Disabilities Research Reviews 5, 125-131.

Ward GR, Huang Y-S, Bobik E, Xing H-C, Mutsaers L, Auestad N, Montalto M \& Wainwright PE (1998) Long-chain polyunstaurated fatty acid levels in formulae influence deposition of docosahexaenoic acid and arachidonic acid in brain and red blood cells of artificially reared neonatal rats. Journal of Nutrition 128, 2473-2487.
Wauben IPM \& Wainwright PE (1999) The influence of neonatal nutrition on behavioral development: A critical appraisal. Nutrition Reviews 57, 35-44.

Werkman SW \& Carlson SE (1996) A randomized trial of visual attention of preterm infants fed docosahexaenoic acid until nine months. Lipids 31, 91-97.

Willatts P, Forsyth JS, DiMondugno MK, Varma S \& Colvin M (1998) Effect of long-chain polyunsaturated fatty acids in infant formula on problem solving at 10 months of age. Lancet $\mathbf{3 5 2}$, 688-691.

Zimmer I, Hembert S, Dward G, Breton P, Guilloteau D, Besnard J-C \& Chalon S (1998) Chronic $n-3$ polyunsaturated fatty acid diet-deficiency acts on dopamine metabolism in the rat frontal cortex: a microdialysis study. Neuroscience Letters 240, $177-181$ 